\title{
Recurrent Aortobronchial Fistula after Endovascular Stenting for Infected Pseudoaneurysm of the Proximal Descending Thoracic Aorta: Case Report
}

\author{
Sun-Geun Lee, M.D. ', Seung Hyong Lee, M.D. ${ }^{2}$, Won Kyoun Park, M.D. ', Dae Hyun Kim, M.D., Ph.D. ', \\ Jae Won Song, M.D. ${ }^{1}$, Sang-Ho Cho, M.D., Ph.D. ${ }^{1}$
}

'Department of Thoracic and Cardiovascular Surgery, Kyung Hee University Hospital at Gangdong, Kyung Hee University School of Medicine; ${ }^{2}$ Department of Thoracic and Cardiovascular Surgery, Kyung Hee University Hospital, Kyung Hee University School of Medicine, Seoul, Korea

\section{ARTICLE INFO}

Received October 12, 2020

Revised November 2, 2020

Accepted November 12, 2020

Corresponding author

Sang-Ho Cho

Tel 82-2-440-6158

Fax 82-2-440-8004

E-mail sinan75@khnmc.or.kr

ORCID

https://orcid.org/0000-0001-5590-1904

Aortobronchial fistula (ABF) induced by an infected pseudoaneurysm of the thoracic aorta is a life-threatening condition. As surgical treatment is associated with significant mortality and morbidity, thoracic endovascular aneurysm repair (TEVAR) may be an alternative for the treatment of ABF. However, the long-term durability of this intervention is largely unknown and the recurrence of $\mathrm{ABF}$ is a potential complication. We experienced a case of recurrent $A B F$ after stent grafting as an early procedure for an infected pseudoaneurysm of the thoracic aorta. Remnant ABF, bronchial and/or aortic wall erosion, vasa vasorum connected with $A B F$, and recurrent local inflammation of the thin aortic wall around $A B F$ might cause recurrent hemoptysis. As a result, we suggest that TEVAR should be considered as a bridge therapy for the initial treatment of $A B F$ resulting from an infected pseudoaneurysm, and that several options, such as second-stage surgery, should be considered to prevent the recurrence of $\mathrm{ABF}$.

Keywords: Aorta, Aneurysm, Infected, Bronchial fistula, Endovascular procedures, Case report

\section{Case report}

A 59-year-old man was admitted to the emergency room following the occurrence of massive hemoptysis with a highgrade fever. The patient's blood pressure was $110 / 70 \mathrm{~mm}$ $\mathrm{Hg}$ and his pulse rate was 100 beats per minute. The patient's clinical data on admission were as follows: white

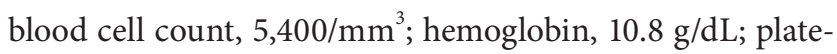
let count, $298 \times 10^{3} / \mathrm{mm}^{3}$; and C-reactive protein, $11.2 \mathrm{mg} /$ $\mathrm{dL}$. One month ago, he had presented to an outpatient clinic to investigate the origin of the fever and oral antibiotics had been administered under the diagnosis of a urinary tract infection (urine culture: Salmonella species). Enhanced computed tomography (CT) of the chest revealed a saccular aortic aneurysm of the proximal descending thoracic aorta (Fig. 1). The urine and sputum culture reported Salmonella species. Initially, under a diagnosis of aortobronchial fistula (ABF) from the infected pseudoaneurysm, we planned to perform open surgery to remove the ABF. However, due to recurrent hemoptysis, emergency stent grafting was performed in order to control the hemoptysis $(36 \times 120 \mathrm{~mm}$, S\&G SEAL; S\&G Biotech Inc., Seoul, Korea). Intravenous antibiotics were given daily for 1 month, according to the guidelines for the management of infective endocarditis, and the patient was then switched to oral antibiotics. The duration of oral antibiotic therapy (levofloxacin) was 60 days and the patient recovered uneventfully. Chest CT on postoperative day 50 confirmed shrinkage of the aneurysm with disappearance of the pulmonary infiltrates surrounding the aneurysmal wall, and there was no endoleak. Approximately 4 months after stent grafting, the patient was readmitted with recurrent mild hemoptysis. The patient's blood pressure was 120/70 mm Hg and his pulse rate was 90 beats per minute. His clinical data on admission were as follows: white blood cell count, 10,600/ $\mathrm{mm}^{3}$; hemoglobin, $11.5 \mathrm{~g} / \mathrm{dL}$; hematocrit, 

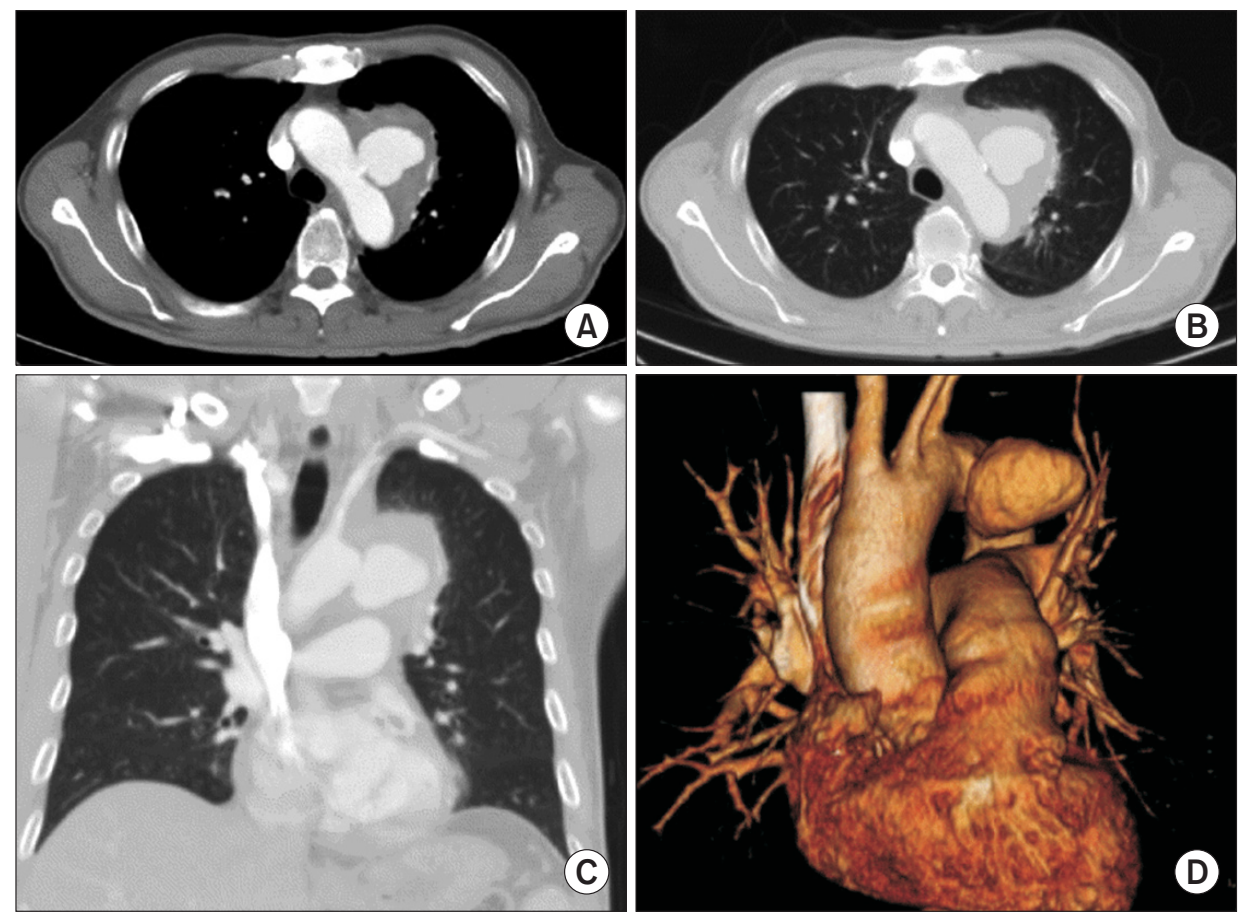

Fig. 1. Initial chest CT. (A, B) Axial CT images show a saccular aortic aneurysm of the proximal descending thoracic aorta. (C) Coronal reconstructed CT image showing the left subclavian artery and the aneurysm. (D) Three-dimensional reconstructed CT image showing the aneurysm. CT, computed tomography.
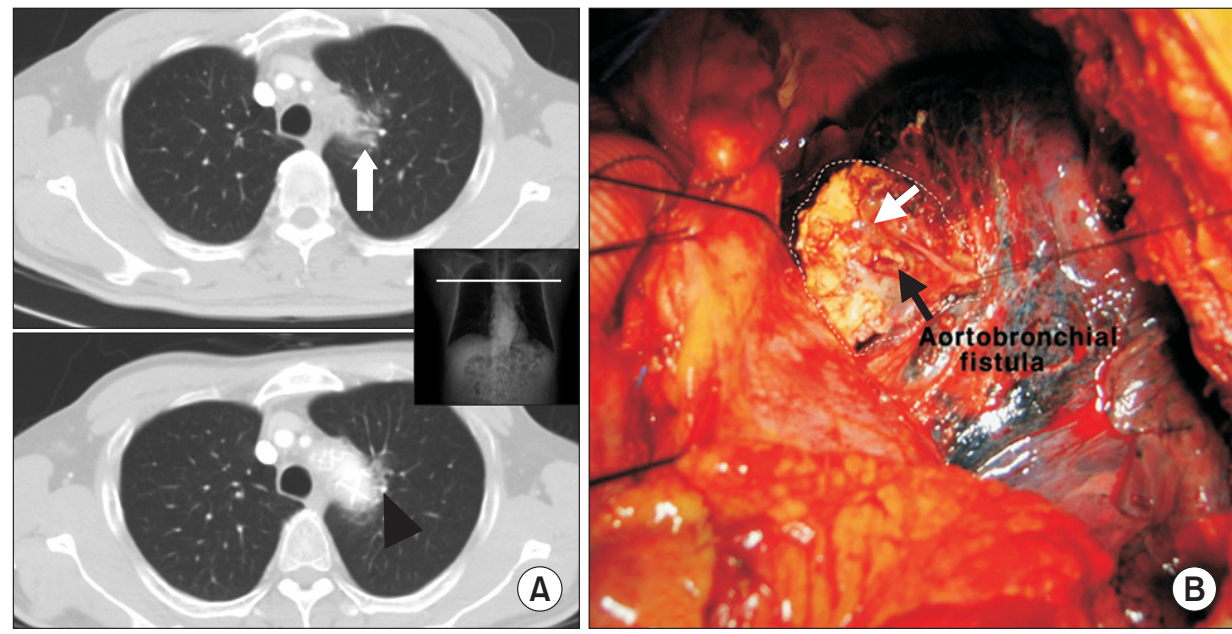

Fig. 2. (A) Follow-up chest computed tomography, 4 months after endovascular stent graft repair. The arrowhead indicates aneurysm sac shrinkage. The arrow point indicates pulmonary infiltrates adjacent to the regressed aortic aneurysm wall. (B) Intraoperative finding of ABF. The white arrow point indicates the thin aortic wall around the ABF. The white dashed line indicates the margin of the aortic wall attached to the lung. The black arrow point indicates the remnant $\mathrm{ABF}$. $\mathrm{ABF}$, aortobronchial fistula.

$32.8 \%$; platelet count, $27.0 \times 10^{4} / \mathrm{mm}^{3}$; and C-reactive protein, $3.5 \mathrm{mg} / \mathrm{dL}$. Chest CT showed pulmonary infiltrates adjacent to the regressed aortic aneurysm wall (Fig. 2A). Bronchoscopy revealed scattered blood clots extending from the left upper lobe to the left main bronchus with no evidence of an active focus of hemoptysis. We performed eradication of all previous infected tissue, including the stent graft, and reconstruction of the distal ascending aorta, aortic arch, and proximal descending thoracic aorta with a woven Dacron graft under cardiopulmonary bypass. Pathological examination of the incised aorta revealed atherosclerosis with acute and chronic inflammation. Howev- er, cultures of the aortic wall and the graft did not detect any bacteria. It would seem that remnant $\mathrm{ABF}$ (Fig. 2B, black arrow), vasa vasorum connected with the $\mathrm{ABF}$, and recurrent local inflammation of the thin aortic wall around the ABF (Fig. 2B, white arrow) might have caused recurrent hemoptysis. After intravenous antibiotics were given daily for 1 month, he was discharged home without any complications and was followed up without the recurrence of $\mathrm{ABF}$ at an outpatient clinic for 80 months.

The patient provided informed consent for the publication of his clinical details and images. 


\section{Discussion}

$\mathrm{ABF}$ is a rare, life-threatening condition that represents a communication between the thoracic aorta and the tracheobronchial airway. An ABF is characterized by massive or recurrent hemoptysis in patients with a history of previously treated thoracic aortic pathology, atherosclerotic aneurysms, traumatic injuries of the descending thoracic aorta, and infected aortic pseudoaneurysm [1,2]. The traditional treatment of $\mathrm{ABF}$ involves thoracotomy and in situ graft replacement or extra-anatomical bypass. Early mortality rates after surgical repair were reported to be $18 \%$ to $24 \%$, with substantial postoperative morbidity [3]. Although early open thoracic surgery under cardiopulmonary bypass is essential, the high mortality and morbidity associated with surgery may lead surgeons to consider other options; in particular, thoracic endovascular aneurysm repair (TEVAR) may be an alternative for the treatment of ABF. This treatment is less invasive and associated with acceptable early mortality and morbidity rates [1,2,4-6]. In 2013, Canaud et al. [4] carried out a systematic review of the outcomes of TEVAR for ABF, reporting 134 cases with excellent short-term results in comparison with the results of open surgery, including a low 30-day mortality rate (5.9\%) and no cases of paraplegia. The incidence of severe complications (pulmonary complications, sepsis, myocardial ischemia) was only $10.4 \%$. In a review of the literature, Riesenman et al. [1] reported a 30-day mortality rate of $1.5 \%$ in 67 patients undergoing TEVAR for ABF.

However, the long-term durability of stent grafts remains unknown. Concerns about the risk of the recurrence of $\mathrm{ABF}$ or infection of the stent graft have been raised [7-9]. Canaud et al. [4] reported that recurrence of ABF and stent graft infections after TEVAR were observed in $11.2 \%$ and $1.7 \%$ of patients, respectively. Surgical conversion was required in $4.3 \%$ of the patients [4-7]. In cases of ABF resulting from an infected pseudoaneurysm, an infectious break in the wall of an artery with the formation of a saccular outpouching and concomitant systemic infection may aggravate the outcomes after TEVAR $[9,10]$. In conclusion, including the patient described here from our institution, a recent body of literature points toward the conclusion that the early outcomes of TEVAR are excellent compared to the outcomes of open surgical intervention; however, the mid-term recurrence rate of $\mathrm{ABF}$ after TEVAR may be not acceptable [3].

Whether local infections and inflammations can be completely resolved when an endoprosthesis is placed within the infected field is unknown. Remnant ABF, bron- chial and/or aortic wall erosion, the vasa vasorum connected with the $\mathrm{ABF}$, and recurrent local inflammation of the thin aortic wall around ABF might cause recurrent hemoptysis $[9,10]$. The images of our present case may demonstrate the potential instability of ABF induced by an infected pseudoaneurysm and the surrounding tissues after TEVAR.

Recent investigations suggest that TEVAR should be followed by delayed, durable open surgery when the patient has recovered and is well enough in terms of hemodynamic and physiological stability $[7,8,10]$. In young and fit patients, the debridement of all infected tissues, graft replacement of the aorta, and flap coverage may be fundamental treatment for a staged operation [10]. In addition, Canaud et al. [7] suggested that pulmonary resection (bronchus and/or lung parenchyma) with coverage of the stent graft using muscle or pleural flaps could be an additional option. The treatment strategy and the intervals until additional procedures are individualized according to the patient's physical condition and severity of sepsis.

Herein, we report a case of recurrent ABF after stent grafting as an early procedure for an infected pseudoaneurysm of the proximal descending thoracic aorta. We suggest that TEVAR should be considered as bridge therapy for the initial treatment of ABF resulting from an infected pseudoaneurysm, and that several options, such as second-stage surgery, should be considered to prevent the recurrence of $\mathrm{ABF}$.

\section{Conflict of interest}

No potential conflict of interest relevant to this article was reported.

\section{ORCID}

Sun-Geun Lee: https://orcid.org/0000-0002-7509-0229

Seung Hyong Lee: https://orcid.org/0000-0002-9839-6431

Won Kyoun Park: https://orcid.org/0000-0002-1693-0582

Dae Hyun Kim: https://orcid.org/0000-0002-8434-7380

Jae Won Song: https://orcid.org/0000-0002-3530-0623

Sang-Ho Cho: https://orcid.org/0000-0001-5590-1904

\section{References}

1. Riesenman PJ, Brooks JD, Farber MA. Thoracic endovascular aortic repair of aortobronchial fistulas. J Vasc Surg 2009;50:992-8.

2. Kawaharada N, Kurimoto Y, Ito T, et al. Endovascular stent-graft repair of aortobronchial fistulas. Ann Thorac Surg 2012;94:524-9. 
3. Nishizawa J, Matsumoto M, Sugita T, et al. Surgical treatment of five patients with aortobronchial fistula in the aortic arch. Ann Thorac Surg 2004;77:1821-3.

4. Canaud L, Ozdemir BA, Bahia S, Hinchliffe R, Loftus I, Thompson M. Thoracic endovascular aortic repair for aortobronchial fistula. Ann Thorac Surg 2013;96:1117-21.

5. Bailey CJ, Force S, Milner R, Kasirajan K, Veeraswamy RK. Thoracic endovascular repair as a safe management strategy for aortobronchial fistulas. J Vasc Surg 2011;53:1202-9.

6. Bozzani A, Arici V, Odero A. Aortic stentgraft in aortobronchial fistula is a bridge solution? Ann Thorac Surg 2013;95:381-2.

7. Canaud L, D'Annoville T, Ozdemir BA, Marty-Ane C, Alric P. Com- bined endovascular and surgical approach for aortobronchial fistula. J Thorac Cardiovasc Surg 2014;148:2108-11.

8. Nguyen T, Peters P, McGahan T, Shah P. Staged management of a primary aortobronchial fistula: a novel approach using a trapezius flap repair. Heart Lung Circ 2012;21:292-4.

9. Touma Y, Demondion P, Rama A, Leprince P. Aortobronchial fistula in the presence of a midgraft hole after endovascular repair of thoracic aortic aneurysms. Eur J Cardiothorac Surg 2013;43:1259-61.

10. De Rango P, Estrera AL, Azizzadeh A, Keyhani K, Safi HJ. Twostage safe repair of aortobronchial fistula. Ann Thorac Surg 2010;89: 275-7. 